\title{
A new score model to predict the inducibility of supraventricular tachycardia in patients with palpitation without documented electrocardiography
}

\author{
Yusuf Türkmen (D), Naci Babat ${ }^{1}$ (D) \\ Department of Cardiology, Karolinska University Hospital, Karolinska Institutet; Stockholm-Sweden \\ ${ }^{1}$ Department of Cardiology, Faculty of Medicine, Yüzüncü Yıl University; Van-Turkey
}

\section{ABSTRACT}

Objective: Although electrophysiological study (EPS) is one of the most useful diagnostic approaches to detect the cardiac origin of palpitations, there is no clear indication for EPS in patients with palpitation in the absence of documented supraventricular tachycardia (SVT). In this study, we aimed to create a new SVT score model to detect the requirement of EPS in patients with palpitation without documented tachycardia.

Methods: A total of 154 patients with palpitation were included in this study. To create the SVT score, we considered four main criteria with their subunits. They were severity, frequency, duration, and termination of the symptoms. EPS was performed on all the patients after their scoring assessment.

Results: Median SVT score of induced tachycardia and non-induced patients was determined as 10 and 6 points, respectively; and it was found to be statistically significant; $p<0.001$. The cut-off value for inducibility was found to be 7.5 points through receiver operating characteristic curve analysis, (94.9\% sensitivity, $92.1 \%$ specificity), and the area under the curve was determined to be $97.2(94.8-99.5, p<0.001)$.

Conclusion: According to our new SVT score model, $\geq 8$ points strongly correlates with the inducibility of SVT during EPS.

Keywords: intracardiac electrophysiology study, palpitations, supraventricular tachycardia score formula, tachycardia inducing

Cite this article as: Türkmen $Y, B a b a t ~ N$. A new score model to predict the inducibility of supraventricular tachycardia in patients with palpitation without documented electrocardiography. Anatol J Cardiol 2021; 25: 646-52.

\section{Introduction}

Palpitation is a sensed abnormality of the heartbeat represented by awareness of heart muscle contractions in the thorax: strong, fast and/or unequal beats, and pauses. It is the most common complaint of patients who are admitted to cardiology clinics $(1,2)$. Palpitation is frequently associated with cardiac and psychiatric disorders $(2,3)$. Although a small amount of palpitations can be life threatening, most of them are associated with a structurally normal heart $(4,5)$. Palpitations can be intermittent or continuous. Accompanying symptoms are dizziness, shortness of breath, sweating, headache, and chest pain (6).

Although palpitations are the most common cause of admissions to cardiology clinics, it is difficult to find the exact cause of palpitations. Initial physical examination, electrocardiography
(ECG) recordings, and laboratory results of patients are usually normal. A 24-hour Holter monitoring and external rhythm recording tests up to 30 days are easy to apply and non-invasive but have very low success rates in the detection of arrhythmias (7). An implantable loop recorder (ILR) can stay in the body for several years and can better detect clinically relevant arrhythmias. However, the high cost of the device restricts the use of ILR $(8,9)$.

Electrophysiological study (EPS) is one of the most useful diagnostic approaches in the detection of cardiac origin of palpitation $(10,11)$. However, requirement of hospitalization, cost, complications, and the concerns of patients about the invasive procedure makes it difficult to perform in all patients with palpitation. Currently, neither guidelines nor clear indications are available about the requirement of EPS in patients with palpitation in the absence of documented supraventricular tachycardia 


\section{HIGHLIGHTS}

- Deciding to perform an electrophysiological study (EPS) owing to the suspicion of supraventricular tachycardia (SVT) in the absence of documented tachycardia is crucial in terms of time and cost.

- Symptom variety, frequency, duration, and termination pattern of SVTs play an important role in the prediction of tachycardia inducibility during EPS.

- Our new score model based on the symptoms of SVT strongly predicts the inducibility of tachycardia during EPS.

(SVT) episodes. To fill this gap and clarify the necessity of EPS in patients with palpitation, we developed an equation which is based on the characteristics of palpitation.

\section{Methods}

A total of 154 patients who were admitted to either emergency or cardiology departments with palpitation between July 2017 and November 2020 were included in this study. Detailed medical histories, blood samples, and ECG recordings of the patients were obtained at their first admission. Although palpitations were present in all the patients, they were in sinus rhythm on admission; however, 13 of them had previously detected SVT on ECG according to the regional ECG recording database. Although the patients were admitted with palpitations, none of them had SVT accompanied by palpitations. Patients with known pregnancy, alcohol consumption in the past 24 hours, severe heart valve diseases, cancer, antiarrhythmic medication intake or patients with a high suspicion of ventricular tachycardia because of wide QRS complexes, known atrial fibrillation (AFib), and all types of atrioventricular block were excluded. SVT was defined as the presence of heart rate over 100 beats/minute with narrow QRS (<100 msec) morphology or presence of wide QRS (>100 msec) according to the SVT discrimination algorithm of Brugada (12). All the four criteria of Brugada were carefully applied to determine wide ORS SVT. The printer speed of 12-lead ECG recording was $25 \mathrm{~mm} / \mathrm{sec}$ with $10 \mathrm{~mm} / \mathrm{mV}$ of amplification. All ECG readings were analyzed by the same cardiologists. The final diagnosis of distinctive arrhythmia was made on the basis of the results of EPS. The protocol of research was approved by
Ethics Committee of the local organization of university, and informed consents were obtained from all the patients before the procedure.

\section{Scoring criteria}

We determined four main criteria to detect the inducibility of SVT. Severity, frequency, duration, and termination of symptoms constituted the column of our new SVT scoring system. The first main criterion was the severity of symptoms. The presence of only palpitation was equal to one point; other symptoms such as sweating, flushing, headache, nausea, dizziness, and eye blurriness along with palpitations were equivalent to two points; three points indicated palpitation with presyncope, syncope, and shortness of breath; frequent urination along with palpitations was rated as four points. The second main criterion was the frequency of symptoms. If the symptoms occurred less than once a year, it was scored one point, several times a year was two points, several times per month was three points, and more than once a week was four points. The third main criterion was the duration of symptoms. The presence of symptoms for less than one minute was scored as one point, duration of symptoms less than one hour was two points; if the symptom continued for several hours, it was scored as three points, and the existence of symptoms for more than a day was four points. The last main criterion was the termination of symptoms. Spontaneously, by perioral medication, intravenous medication, and vagal maneuver termination of symptoms were scored as one, two, three, and four points, respectively. Table 1 summarizes all the parameters mentioned above. All the patients were evaluated by same cardiologists. And the scoring criteria mentioned above was used to get the total scores. Electrophysiologists who performed the EPS were blinded to the SVT score of the patients before the procedure.

\section{Electrophysiology study protocol}

EPS was performed only under local anesthetic without administration of intravenous analgesics or sedatives. We placed steerable hexapolar (Livewire ${ }^{\mathrm{TM}}$, Electrophysiology Catheters, Abbott, Plymouth, MN 55442, USA), steerable decapolar (Inquiry ${ }^{\top M}$, Electrophysiology Catheters, Abbott, Plymouth, MN 55442, USA), and fixed curve quadripolar (SupremeTM, Electrophysiology Catheters, Abbott, Plymouth, MN 55442, USA) catheters in the his region, coronary sinus (CS), and right ventricular apex, respectively. Programmed extra stimulus (PES) was performed via CS catheter up to triple extrastimuli to induce

Table 1. Supraventricular tachycardia inducibility score model parameters

\begin{tabular}{|c|c|c|c|c|c|c|c|}
\hline \multicolumn{2}{|c|}{ 1- Symptoms/score } & \multicolumn{2}{|c|}{ 2-Frequency/score } & \multicolumn{2}{|c|}{ 3-Duration/score } & \multicolumn{2}{|c|}{ 4-Termination/score } \\
\hline Palpitation & $=1$ & $<1 /$ Year & $=1$ & $<1$ Minute & $=1$ & Spontaneously & $=1$ \\
\hline +Sweating... & $=2$ & $>1 /$ Year & $=2$ & $<1$ Hour & $=2$ & PO medication & $=2$ \\
\hline +Syncope... & $=3$ & $>1 /$ Month & $=3$ & $>1$ Hour & $=3$ & IV medication & $=3$ \\
\hline +Urination & $=4$ & >1/Week & $=4$ & >1 Day & $=4$ & Vagal maneuvers & $=4$ \\
\hline
\end{tabular}




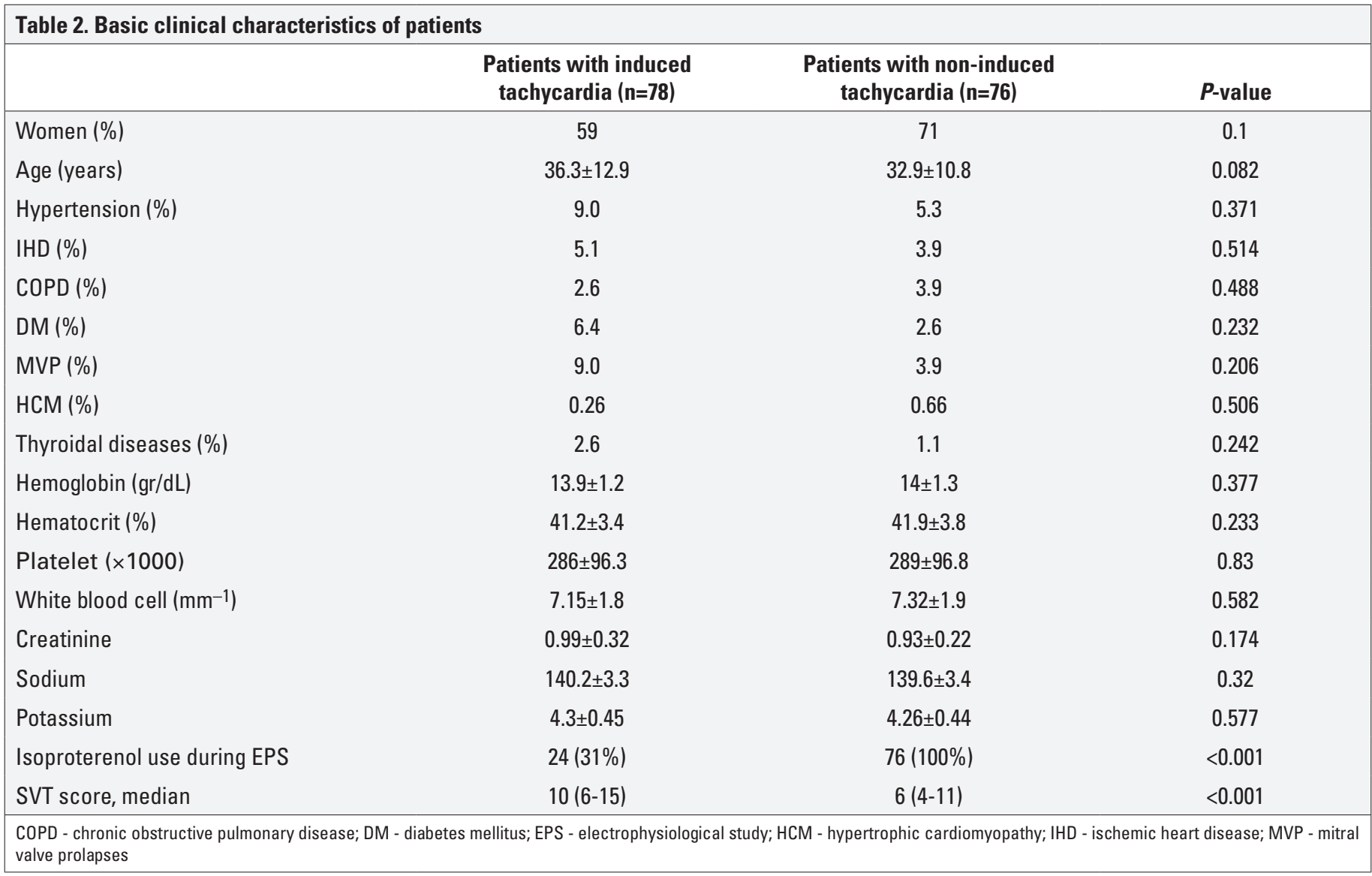

arrhythmias. Concentric or eccentric activation of retrograde conductions were recorded. Patients without any inducible tachycardia with only PES received intravenous $20 \mathrm{mcg}$ bolus of isoproterenol, followed by $2 \mathrm{mcg} / \mathrm{min}$ infusion and titrated up to $20 \mathrm{mcg} / \mathrm{min}$ according to the patient's response. The desired increase in heart rate during isoproterenol infusion was at least $30 \%$. Thereafter, stimulations were repeated from the same site under isoproterenol infusion. All the SVT discrimination criteria were considered to get a clear and detailed diagnosis of the tachycardia. Right ventricular (RV) pacing was consciously avoided so as to not induce any VT as a cause of palpitation because our aim was to induce SVT instead of VT. Therefore, we did not perform PES from RV to induce any type VT to discover the reason of palpitation. All types of induced SVTs, except three left-sided atrial tachycardia (AT) and eight AFib, were treated by radiofrequency (RF) or focal cryoablation techniques. Pulmonary vein isolation and the treatment of left-sided ATs were postponed owing to the possible high risk of thrombosis in the left atrium and absence of transesophageal echocardiography examination before the procedure. The ablation of slow pathway in the presence of atrioventricular nodal reentrant tachycardia (AVNRT) was performed by $6 \mathrm{~mm}$-tip focal cryocatheter (Medtronic Inc, Minneapolis, MN, USA). All other SVTs were cured by RF energy. After successful ablation, we waited at least 20 minutes and repeated the PES from appropriate sites to induce the clinical tachycardia. Two cases of AVNRT and two cases of atrioventricular reentrant tachycardia (AVRT) were induced again, and they were successfully re-ablated.

\section{Laboratory tests}

Complete blood count, urea, creatinine, sodium, potassium, thyroid stimulating hormone (TSH), C-reactive protein (CRP), and glucose serum levels of patients were evaluated on the first admission of patients. Transthoracic echocardiography was performed on all the patients to evaluate the functional and structural state of heart. Two patients with moderate to severe aortic insufficiency, three with severe tricuspid regurgitation, and five with either mitral valve insufficiency or stenosis were excluded. The TSH values were found to be $<0.001 \mathrm{mIU} / \mathrm{L}$ in two patients, one of whom was diagnosed with Hashimoto's thyroiditis. Her main complaint was frequent and spontaneously terminated palpitation with flushing and sweating symptoms. The CRP levels in eleven patients were $>5 \mathrm{mg} / \mathrm{L}$, but they had normal body temperature. There was no electrolyte disturbance in any of the patients.

\section{Holter monitoring}

No patient underwent rhythm Holter monitoring after their last admission. They were informed about the study and immediately admitted to the hospital with their consent. They also did not undergo telemetry monitoring before the procedure because of the low risk of serious arrhythmia. However, 22 patients had already under- 


\begin{tabular}{|c|c|c|c|c|c|c|c|}
\hline & Accuracy & Sensitivity & Specificity & +PDV & -PDV & +LHR & -LHR \\
\hline \multicolumn{8}{|c|}{ Symptom } \\
\hline$>1$ & $72 \%(65-79)$ & $97.4 \%$ (91-99.7) & $47.4 \%(35.8-59.1)$ & $65.5 \%(60.5-70.2)$ & $94.7 \%(81.8-98.6)$ & $1.85(1.49-2.30)$ & $0.05(0.01-0.22)$ \\
\hline$>2$ & $71 \%(63-78)$ & $50 \%(38.5-61.5)$ & $92,1 \%(83.6-97)$ & $86.7 \%(74.5-93.5)$ & $64.2 \%(58.7-69.3)$ & $6.33(2.85-14.09)$ & $0.54(0.43-0.68)$ \\
\hline \multicolumn{8}{|c|}{ Duration } \\
\hline$=1$ & $28 \%(21-36)$ & $2.6 \%(0.3-8.9)$ & $54 \%(42.1-65.4)$ & $5.4 \%(1.4-16.6)$ & $35 \%(30.4-39.9)$ & $0.06(0.01-0.22)$ & $1.81(1.46-2.23)$ \\
\hline$>1$ & $72 \%(64-79)$ & $97.4 \%$ (91-99.7) & $46 \%(34.6-57.9)$ & $65 \%(60-69.6)$ & $94.6 \%(81.4-98.6)$ & $1.81(1.46-2.23)$ & $0.06(0.01-0.22)$ \\
\hline$>2$ & $72 \%(64-79)$ & $47.4 \%(36-59)$ & $97 \%(90.8-99.7)$ & $94.9 \%(82.2-98.7)$ & $64.3 \%(59.3-69)$ & $18.03(4.5-72.18)$ & $0.54(0.44-0.67)$ \\
\hline$>1$ & $74 \%(66-81)$ & $94.9 \%(87.4-98.6)$ & $39.5 \%(28.4-51.3)$ & $61.7 \%(57.1-66)$ & $88.2 \%(73.5-95.3)$ & $1.57(1.30-1.89)$ & $0.13(0.05-0.35)$ \\
\hline$>2$ & $83 \%(76-89)$ & $70.5 \%(59.1-80.3)$ & $96 \%(88.9-99.2)$ & $94.8 \%(85.7-98)$ & $76 \%(69.2-81.8)$ & $17.86(5.84-54)$ & $0.31(0.22-0.43)$ \\
\hline$>3$ & $55 \%(46-63)$ & $10.3 \%(4.5-19.2)$ & $100 \%(95.3-100)$ & $100 \%(59.7-100)$ & $52 \%(50.2-53.9)$ & $\approx$ & $0.90(0.83-0.97)$ \\
\hline \multicolumn{8}{|c|}{ Termination } \\
\hline$=1$ & $23 \%(17-31)$ & $41 \%(30-52.8)$ & $5.3 \%(1.5-13)$ & $30.8 \%(25.3-37)$ & $8 \%(3.2-18.7)$ & $0.43(0.33-0.57)$ & $11.2(4.2-29.6)$ \\
\hline$>1$ & $77 \%(69-83)$ & $58.9 \%(47.2-70)$ & $94.7 \%(87-98.6)$ & $92 \%(81.3-96.8)$ & $69.2 \%(63.2-74.7)$ & $11.2(4.2-29.6)$ & $0.43(0.33-0.57)$ \\
\hline$>2$ & $70 \%(62-77)$ & $42.3 \%(31.2-54)$ & $100 \%(92.3-100)$ & $97 \%(82.2-99.6)$ & $62.5 \%(57.9-66.9)$ & $32.1(4.5-229.2)$ & $0.58(0.48-0.71)$ \\
\hline$>3$ & $67 \%(59-74)$ & $34.6 \%(24.2-46.2)$ & $100 \%(95.2-100)$ & $100 \%(84.5-100)$ & $59.8 \%(55.9-63.7)$ & $\approx$ & $0.65(0.56-0.77)$ \\
\hline
\end{tabular}

gone Holter monitoring earlier because of their palpitations. Holter recordings showed some signs of SVT in four patients, but they were not satisfactory enough to make a $100 \%$ correct diagnosis.

\section{Statistical analysis}

All statistical analyses were performed using the Statistical Product and Service Solutions version 20 for Windows (SPSS Inc., Chicago, IL, USA) or BDT comparator (13). The data were tested with the Kolmogorov-Smirnov test to see if they met the normal distribution or not. Numerical data corresponding to normal distribution were expressed as mean \pm standard deviation, and numerical data that did not correspond to normal distribution were expressed as median (minimum-maximum). Categorical variables were presented as counts and percentages. Basic characteristics were compared with the chi-squared test, independent sample t-test, or Mann-Whitney U test when needed. The performance of binary decision rules was described using the following measures [with $95 \%$ confidence interval $(\mathrm{Cl})$ ]: diagnostic accuracy, sensitivity, specificity, positive predictive value, negative predictive value, likelihood ratio of a positive test $(\mathrm{LR}+$ ), and negative test (LR-). Performance of the 'SVT score' in discrimination of SVT induced and non-induced patients was assessed by using the receiver operating characteristic $(\mathrm{ROC})$ curve and area under the ROC curve. $P$ values $<0.05$ were considered as statistically significant.

\section{Results}

In our study, SVT was inducible in 78 of the total 154 patients. The average age of SVT induced and non-induced patients were $36.3 \pm 12.9$ and $32.9 \pm 10.8$ years, respectively. The ratio of women in both groups was higher than men $(59.2 \%$ of patients in inducible and $71.8 \%$ in non-inducible group), but it was not statistically significant $(p=0.12)$. Other demographic features are shown in Table 2 . Forty-seven $(60.2 \%)$, fifteen $(19.2 \%)$, eight $(10.3 \%)$, five $(6.4 \%)$, and three $(3.9 \%)$ of all tachycardia induced patients were diagnosed with AVNRT, AVRT, AFib, AT, and atrial flutter, respectively, on the basis of the results of EPS. Median SVT scores of tachycardia induced and non-induced patients were 10 and 6 points, respectively, and it was found to be statistically significant, $p<0.001$. SVT scores of tachycardia subtypes were similar to each other and were not statistically significant. Three patients with non-induced tachycardia were diagnosed with inappropriate sinus node tachycardia according to EPS and rhythm Holter recordings. Their SVT scores were 6, 6, and 7 points, respectively. Two patients had monomorphic ventricular extra systole (VES) during EPS, and both of them clearly described palpitations in connection with extra beats and were excluded from the study owing to a high suspicion of VES related symptoms. In addition, all short-lived ( $<10 \mathrm{sec})$ and self-terminated tachycardias such as AT or fibrillation were not accepted as EPS-inducible tachycardia. 


\begin{tabular}{|lcccccccccccc|}
\hline Table 4. Distribution of SVT scores in tachycardia induced and non-induced groups of patients & 10 \\
\hline Diagnosis/SVT scores & $\mathbf{4}$ & $\mathbf{5}$ & $\mathbf{6}$ & $\mathbf{7}$ & $\mathbf{8}$ & $\mathbf{9}$ & $\mathbf{1 0}$ & $\mathbf{1 1}$ & $\mathbf{1 2}$ & $\mathbf{1 3}$ & $\mathbf{1 4}$ & $\mathbf{1 5}$ \\
\hline Number of patients with induced tachycardia & 0 & 0 & 1 & 3 & 12 & 18 & 11 & 6 & 10 & 9 & 7 & 1 \\
Number of patients with non-induced tachycardia & 6 & 24 & 30 & 10 & 3 & 2 & 0 & 1 & 0 & 0 & 0 & 0 \\
Percentage of SVT inducibility according to the scores & 0 & 0 & 3.2 & 23.1 & 80 & 90 & 100 & 85.7 & 100 & 100 & 100 & 100 \\
\hline SVT - supraventricular tachycardia & & & & & &
\end{tabular}

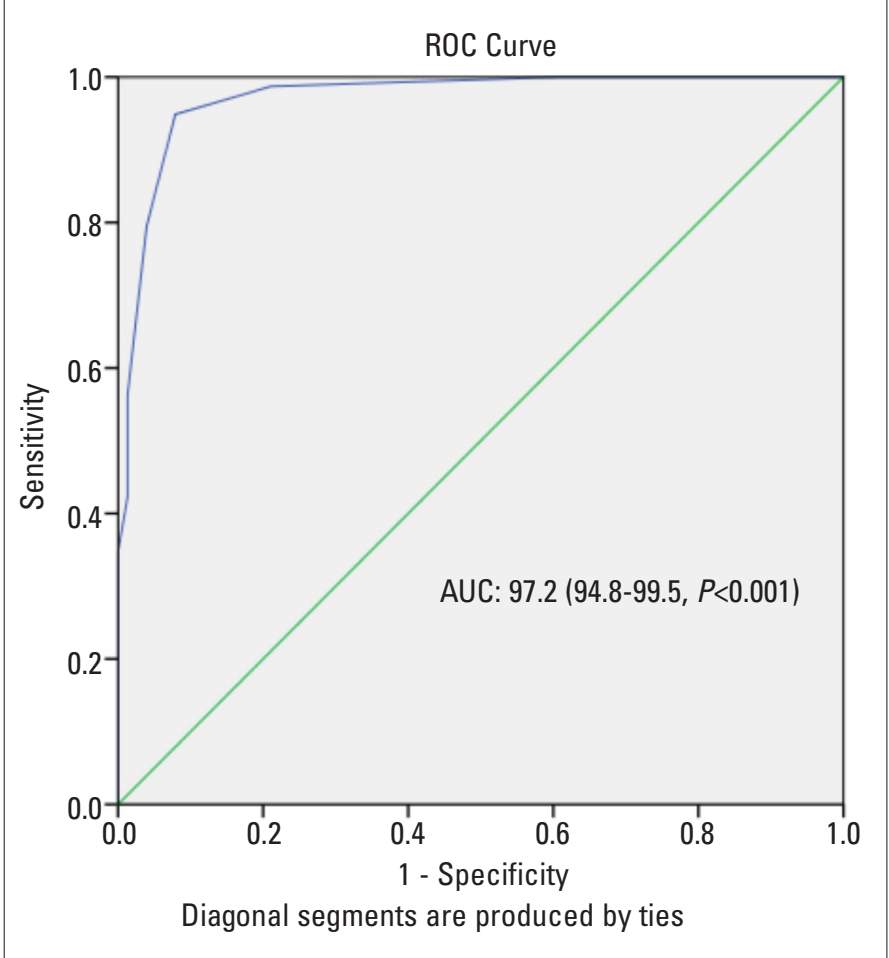

Figure 1. ROC analysis for scoring system to detect SVT inducibility. Threshold score to detect SVT inducibility is 7.5 (sensitivity, 94.9\%; specificity, 92.1\%)

AUC - area under the curve; ROC - receiver operating characteristic; SVT supraventricular tachycardia

When the diagnostic performance values of each component of the scoring system were analyzed in patients with induced tachycardia, $\geq 3$ points for each component of SVT scoring system had a high specificity value in the majority of patients (Table 3). The cut-off value for SVT inducibility was found to be 7.5 points (94.9\% sensitivity, $92.1 \%$ specificity). As shown by ROC analysis, the area under curve (AUC) was determined as 97.2 $(94.8-99.5, p<0.001)$ in patients with induced tachycardia (Fig. 1). When the total SVT score was 8 points, the inducibility probability of SVT during EPS was almost four times higher than when it was 7 points (Table 4). We could not induce any type of tachycardia when the SVT score was $\leq 5$ and could induce at least one kind of SVT in all the patients when the SVT score was $>11$ points. When we analyzed the diagnostic performance index of the SVT scoring system, we observed that values $>7$ points were related to significantly high probability of tachycardia inducibility, and scores $\geq 12$ had $100 \%$ inducibility ratio in terms of SVT inducibility and yielded no false positive result with this new SVT scoring system (Table 4).

\section{Discussion}

Although ECG based diagnostic algorithms have been described in the literature (14), we needed a symptom based diagnostic algorithm or scoring system to guess the inducibility of SVT during EPS in patients with a suspicion of SVT. According to our new scoring system, when the total score is $\geq 8$, the inducibility rate of SVT during EPS increases significantly. We were able to induce at least one type of SVT in $50.6 \%$ of our patients, and $60 \%$ of them were AVNRT. Vallès et al. (10) have also induced some kind of SVT in $50 \%$ of their patients with palpitation. In addition, long duration, feeling in the neck, and sudden termination of palpitation were deeply associated with SVT inducibility. In their study, the most induced SVT was AVNRT with a $50 \%$ frequency. Lauschke et al. (15) evaluated patients with palpitation in the absence of documented tachycardia. They were also able to induce at least one type of SVT in $63.7 \%$ of patients, and $55.9 \%$ patients with induced tachycardia had AVNRT. Of note, 13 patients already had documented narrow QRS tachycardia before EPS, and all of them became inducible either by only PES or PES+ isoproterenol infusion during the procedure. AH jump was detected in the majority of patients, particularly just before AV node refractoriness. Subsequently, detailed analysis of whether this $\mathrm{AH}$ prolonging belonged to a true jump, a bundle branch block, or an accessory pathway that made a latent antidromic transmission was not performed in patients in whom tachycardia could not be induced. However, when echo beat occurred, all aggressive stimulation programs under medication and without medication were performed to induce AVNRT. Even if tachycardia could not be induced, the modification of slow pathway was performed after informing the patients who were very symptomatic or who had discontinued antiarrhythmic therapy only two days ago.

The median SVT score of patients with induced tachycardia was 10 points, and we could not induce any type of tachycardia when the SVT score was below 6 points. SVT was induced in all the patients when the SVT score was 12 points. Termination of tachycardia by intravenous medication or vagal maneuver had $100 \%$ of specificity for inducibility. These patients already had documented ECG recordings before EPS. Some parameters such as frequent urination along with palpitation, daily sensation of palpitation, and its existence for more than one day also had $100 \%$ specificity. However, we believe that even though they have $100 \%$ specificity, they are not clinically valid in all SVT types, except Afib or AT, and they become more significant when evaluated together with other parameters. The frequency of symptoms is absolutely crucial in determining the severity of the 
tachycardia and the treatment strategy. It has been stated in previous studies that the frequency of palpitations varies according to the type of arrhythmias (16). As the aim of our study was to increase the predictability power of the equation by including the possible criteria into the equation, the "frequency" criterion was also included in the formula. Although the duration of episodes is variable, ranging from seconds to 72 hours, it is not certain that such a long period of tachycardia is related to the definitive diagnosis of tachycardia. When the "duration" of symptoms was analyzed, we detected only three patients with palpitations longer than 24 hours. Afib was induced in two of those three patients during EPS, and one was a 31-year-old woman with crista terminalis originated focal AT. Considering cardiomyopathies caused by long-term tachycardias, we believe that such long-term complaints should be considered among the SVT characteristics, although it is unlikely.

Although presyncope and syncope are serious symptoms, they were not matched to high scores because of their nonspecificity. One of the most useful studies regarding syncope and SVT was conducted by Brembilla-Perrot et al. (17). They have clearly shown that normal EPS results in the presence of syncope and palpitation. Locati et al. (8) have also followed 395 patients with palpitation or syncope after implantation of a loop recorder. The four-week diagnostic performance of the loop recorder showed syncope $24.5 \%$ of the time; however, SVT was the reason only $7.3 \%$ of the time in all their patients, which explains why in our study, syncope did not match with higher scores. However, the same study also revealed that recurrent or frequent palpitations were predictors of supraventricular arrhythmias. Recurrent palpitations were reported in $68.4 \%$ patients in their study. Therefore, frequency of recurrence of palpitation was another crucial parameter in our scoring system to detect the inducibility of tachycardia. According to our scoring system, the presence of a few palpitations in a week was related to $70.5 \%$ sensitivity and $96 \%$ specificity to detect the inducibility of tachycardia.

Elevated levels of high-sensitive troponin and atrial natriuretic peptide (ANP) at SVT are well known (18-20). We did not consider troponin, but increased levels of ANP is strongly related to frequent urination after the onset of SVT, particularly in the presence of longstanding episodes. According to Yetkin et al. (21), $45 \%$ of their patients have reported polyuria after SVT. A certain proportion of our patients also described frequent urination after palpitation, and there was $100 \%$ specificity for inducibility of SVT with frequent urination and palpitation. However, this invaluable criterion scores only 4 points and does not imply that the presence of related symptom predicts $100 \%$ inducibility. Its value increases when assessed with other symptoms, and the score reaches 8 points in total, which was the critical threshold for inducibility of SVT.

The final criterion was the termination of palpitation by vagal maneuver or perioral or intravenous medication, which usually indicated cardiac arrhythmias. The patients are usually not familiar with vagal maneuver, which means that it decreases the placebo effect of the approach. Similarly, they can easily feel the sudden termination of the tachycardia after a vagal maneuver. However, perioral medications may start to affect the rhythm after several minutes or hours, and many patients expect to see an effect of the perioral or intravenous medication, even if it is a placebo effect. The worst predictive value was with spontaneous termination of tachycardia because of its very subjective feature. Therefore, spontaneously terminated palpitation scored only 1 point and others higher points. When the specificity rate of spontaneously terminated palpitation was $5.3 \%$ (1 point), it sharply increased to $94.5 \%$ with perioral medication ( 2 points). The presence of anamnesis about the termination of SVT by vagal maneuver or intravenous medication had an extremely high sensitivity rate. Although both of them are highly sensitive parameters, it is worthwhile to compare intravenous medication and vagal maneuver in the termination of tachycardia because of different scores. Intravenous adenosine and beta blockers are used widely, and they are more effective against sinus tachycardia than vagal maneuvers. The broad effective spectrum of intravenous medication decreases its specificity in the detection of real SVT. Therefore, it scored 3 points, instead of 4 . Moreover, some patients have an anticipation during the vascular access and drug administration procedure; however, the placebo effect of vagal maneuver is less as they do not know much about it. Therefore, as vagal maneuver is less effective than intravenous drugs in distinguishing sinus tachycardia, inappropriate sinus tachycardia, or sinus node reentrant tachycardias from true SVTs, its specificity is high in the detection of true SVT. In addition, as some patients have been trained in vagal maneuvering earlier, they can clearly describe the effect of the technique. However, no such intervention was performed by us on any patient we recruited to the study, and it was found that they were subjected to such procedures according to their medical history and records.

\section{Study limitations}

One limitation of our study was that caffeine consumption, energy drinks, smoking, and drug abuse in patients were not examined in detail. Instead of doing blood tests, they were just asked questions about these, and we did not have any clear data regarding how these agents could interfere with our results. In addition, the blood level of catecholamines and other sympathomimetic agents were not analyzed before the procedure. The sudden onset/off phenomenon is one of the most typic features of regular reentrant tachycardia and should be included as a criterion; however, there was no appropriate scoring section for it. The small sample size and subjectivity in symptoms were also the limitations of this study. Therefore, we need further larger and more detailed studies to support and improve the validity of this new scoring model.

\section{Conclusion}

To the best of our knowledge, this is the first scoring trial in the prediction of inducibility of SVT. In our study, we demonstrated the concrete relationship between this new SVT score 
and the inducibility of tachycardia. We hope and believe that this study will be a reference point for other SVT scoring models and shows the necessity of EPS in patients with palpitation in the absence of documented ECG.

Acknowledgments: We are grateful to the meticulous physicians of our emergency department in the detection and evaluation of patients.

Conflict of interest: None declared.

Peer-review: Externally peer-reviewed.

Author contributions: Concept - Y.T., N.B.; Design - Y.T., N.B.; Supervision - Y.T., N.B.; Fundings - None; Materials - Y.T., N.B.; Data collection \&/or processing - Y.T., N.B.; Analysis \&/or interpretation Y.T., N.B.; Literature search - Y.T., N.B.; Writing - Y.T., N.B.; Critical review - Y.T., N.B.

\section{References}

1. Weber BE, Kapoor WN. Evaluation and outcomes of patients with palpitations. Am J Med 1996; 100: 138-48. [Crossref]

2. Jonsbu E, Dammen T, Morken G, Martinsen EW. Patients with noncardiac chest pain and benign palpitations referred for cardiac outpatient investigation: a 6-month follow-up. Gen Hosp Psychiatry 2010; 32: 406-12. [Crossref]

3. Alijaniha F, Noorbala A, Afsharypuor S, Naseri M, Fallahi F, Mosaddegh $\mathrm{M}$, et al. Relationship Between Palpitation and Mental Health. Iran Red Crescent Med J 2016; 18: e22615. [Crossref]

4. Abbott AV. Diagnostic approach to palpitations. Am Fam Physician 2005; 71: 743-50.

5. Price JR. Medically unexplained physical symptoms. Psychiatric Aspects of General Medicine 2008; 36: p449-51. [Crossref]

6. Yetkin E. Symptoms in supraventricular tachycardia: Is it simply a manifestation of increased heart rate? Med Hypotheses 2016; 91: 42-3. [Crossref]

7. de Asmundis C, Conte G, Sieira J, Chierchia GB, Rodriguez-Manero $\mathrm{M}$, Di Giovanni G, et al. Comparison of the patient-activated event recording system vs. traditional $24 \mathrm{~h}$ Holter electrocardiography in individuals with paroxysmal palpitations or dizziness. Europace 2014; 16: 1231-5. [Crossref]

8. Locati ET, Moya A, Oliveira M, Tanner H, Willems R, Lunati M, et al. External prolonged electrocardiogram monitoring in unexplained syncope and palpitations: results of the SYNARR-Flash study. Europace 2016; 18: 1265-72. [Crossref]
9. Quan KJ. Palpitation: Extended Electrocardiogram Monitoring: Which Tests to Use and When. Med Clin North Am 2019; 103: 78591. [Crossref]

10. Vallès E, Martí-Almor J, Bazan V, Suarez F, Cian D, Portillo L, et al. Diagnostic and prognostic value of electrophysiologic study in patients with nondocumented palpitations. Am J Cardiol 2011; 107: 1333-7. [Crossref]

11. Giada F, Raviele A. Clinical Approach to Patients with Palpitations. Card Electrophysiol Clin 2018; 10: 387-96. [Crossref]

12. Brugada P, Brugada J, Mont L, Smeets J, Andries EW. A new approach to the differential diagnosis of a regular tachycardia with a wide ORS complex. Circulation 1991; 83: 1649-59. [Crossref]

13. Fijorek K, Fijorek D, Wisniowska B, Polak S. BDTcomparator: a program for comparing binary classifiers. Bioinformatics 2011; 27: 3439-40. [Crossref]

14. Buttà C, Tuttolomondo A, Di Raimondo D, Milio G, Miceli S, Attanzio $M T$, et al. Supraventricular tachycardias: proposal of a diagnostic algorithm for the narrow complex tachycardias. J Cardiol 2013; 61: 247-55. [Crossref]

15. Lauschke J, Schneider J, Schneider R, Nesselmann C, Tischer T, Glass A, et al. Electrophysiological studies in patients with paroxysmal supraventricular tachycardias but no electrocardiogram documentation: findings from a prospective registry. Europace 2015; 17: 801-6. [Crossref]

16. Wood KA, Drew BJ, Scheinman MM. Frequency of disabling symptoms in supraventricular tachycardia. Am J Cardiol 1997; 79: 145-9. [Crossref]

17. Brembilla-Perrot B, Sellal JM, Olivier A, Villemin T, Manenti $V_{1}$ Brembilla $A$, et al. Electrophysiological study generally is negative in patients $<40$ years suspected of supraventricular tachycardia but also complaining of chest pain and/or syncope. Int $\mathrm{J}$ Cardiol 2016; 203: 1109-13. [Crossref]

18. Jenab $Y$, Ghaffari-Marandi N. Changes of high-sensitive troponin level in a patient with paroxysmal supraventricular tachycardia. Anatol J Cardiol 2012; 12: 77-8. [Crossref]

19. Kuo JY, Wang AM, Chang SH, Hung CL, Chen CY, Shih BF, et al. Responses of cardiac natriuretic peptides after paroxysmal supraventricular tachycardia: ANP surges faster than BNP and CNP. Am J Physiol Heart Circ Physiol 2016; 310: H725-31. [Crossref]

20. Tan SY, Nolan J, Craig K, Swainson CP. Supraventricular tachycardia, right atrial pressure, atrial natriuretic peptide and polyuria--a necessary sequence? J Intern Med 1993; 233: 415-7. [Crossref]

21. Yetkin $E$, Ozturk S, Cuglan B, Turhan $H$. Clinical presentation of paroxysmal supraventricular tachycardia: evaluation of usual and unusual symptoms. Cardiovasc Endocrinol Metab 2020; 9: 153-8. [Crossref] 